\title{
Flora do Rio de Janeiro: Nymphaeaceae
}

\author{
Flora of Rio de Janeiro: Nymphaeaceae
}

\author{
Andréia Donza Rezende Moreira ${ }^{1,2}$ \& Claudia Petean Bove
}

\begin{abstract}
Resumo
O presente estudo tem como objetivo contribuir para o conhecimento das espécies de Nymphaeaceae ocorrentes no estado do Rio de Janeiro. O trabalho tem por base a análise morfológica de materiais depositados em herbários e coletas de campo, além da compilação de dados de literatura. Foram registrados um gênero e seis espécies: Nymphaea amazonum, Nymphaea caerulea, Nymphaea lasiophylla, Nymphaea lingulata, Nymphaea pulchella, Nymphaea rudgeana, das quais Nymphaea lasiophylla é citada pela primeira vez no Rio de Janeiro. Chave para identificação, descrições, ilustrações, dados sobre hábitat, fenologia e distribuição geográfica das espécies são apresentados.
\end{abstract}

Palavras-chave: Nymphaea, florística, planta aquática, taxonomia.

\begin{abstract}
This study focuses on the Nymphaeaceae found in the state of Rio de Janeiro. It is based on a morphological analysis of herbaria collections, as well as on a review of the relevant literature and on collections in the field. A total of one genus and six species were recorded for the state: Nymphaea amazonum, Nymphaea caerulea, Nymphaea lasiophylla, Nymphaea lingulata, Nymphaea pulchella, Nymphaea rudgeana, of which Nymphaea lasiophylla is new record for Rio de Janeiro. An identification key, descriptions, illustrations, habitat data, phenology, and species distribution are also provided.
\end{abstract}

Key words: Nymphaea, floristic inventories, aquatic plant, taxonomy.

\section{Nymphaeaceae Salisb.}

Ervas aquáticas anuais ou perenes, rizomatosas. Folhas alternas, simples, flutuantes, emergentes ou submersas; pecíolo cilíndrico longo, glabro ou pubescente, aerenquimatoso; limbo elíptico-sagitado a orbicular ou peltado, margens inteiras ou denteadas, bordos erguidos ou não, superfície abaxial e pecíolos às vezes revestidos com espinhos. Flores solitárias, flutuantes ou emersas, longo-pedunculadas, antese diurna ou noturna, odoríferas, perfeitas, actinomorfas. Sépalas 4-9, livres; pétalas numerosas (3 a muitas), multisseriadas; transição pétala-estame gradual ou abrupta; estames numerosos (8 a muitos), livres, multisseriados, estames externos às vezes petalóides, internos filiformes; ovário súpero a ínfero, carpelos 3-50, livres ou fusionados lateralmente, formando raios estigmáticos com apêndices carpelares terminais. Fruto cápsula carnosa de deiscência irregular; sementes numerosas, ovóides a globosas, às vezes ariladas.
Nymphaeaceae têm distribuição cosmopolita, possui seis gêneros e 55 espécies (Padget \& Les 2004), além de numerosos híbridos e variedades (Feres \& Amaral 2003). Muitos gêneros são cultivados no mundo todo como ornamentais. No Brasil ocorrem dois gêneros: Nymphaea L. e Victoria Lindl. e 23 espécies (BFG 2015). No estado do Rio de Janeiro ocorre um gênero e seis espécies.

\section{Nymphaea L.}

Folhas geralmente flutuantes; limbo sagitado a orbicular profundamente partido na base, venação radial. Flores entomófilas grandes, emergentes, diurnas ou noturnas; sépalas 4, verdes; pétalas numerosas (7-40), brancas, amarelas, rosas, azuis, vermelhas ou roxas, multisseriadas; estames numerosos (20-700) laminares, geralmente verdes, multisseriados; ovário semi-ínfero, superfície estigmática discóide, com apêndices carpelares triangulares, lineares, lingulados ou claviformes.

\footnotetext{
${ }^{1}$ Universidade Federal do Rio de Janeiro, Depto. Botânica, Museu Nacional, 20940-040, São Cristóvão, Rio de Janeiro, RJ, Brasil.

${ }^{2}$ Autor para correspondência: andreiadonza@hotmail.com
} 
Gênero com aproximadamente 47 espécies de distribuição cosmopolita (Löhne et al. 2007). Possui cinco subgêneros (Conard 1905) dos quais apenas dois, Hydrocallis (neotropical) e Brachyceras (pantropical), ocorrem no Brasil e no Rio de Janeiro. Diferenciam-se basicamente por apresentarem flores noturnas com carpelos de paredes unidas lateralmente (Hydrocallis) e flores diurnas com carpelos de paredes completamente unidas (Brachyceras). Crescem em habitats aquáticos lênticos de água doce ou salobra, ricos em matéria orgânica. No Brasil está representado por 18 espécies nativas ou subespontâneas. No estado do Rio de Janeiro são encontradas seis espécies.

\section{Chave para identificação das espécies}

1. Presença de anel de tricomas no ápice do pecíolo

1. Nymphaea amazonum

1'. Ausência de anel de tricomas no ápice do pecíolo.

2. Apêndices carpelares clavados, transição pétala-estame gradual

6. Nymphaea rudgeana

2'. Apêndices carpelares não clavados, transição pétala-estame abrupta.

3. Antese diurna; apêndices carpelares triangulares.

4. Pétalas brancas

5. Nymphaea pulchella

4'. Pétalas de base branca ou amarela e ápice lilás a lilás-azulado

2. Nymphaea caerulea

3'. Antese noturna; apêndices carpelares lingulados.

5. Limbo de superfície abaxial verde-vinoso com máculas vinosas, venação reticulando fracamente em direção ao ápice, nervuras não impressas; pétalas de 19 a 20 ....... 3. Nymphaea lasiophylla

5'. Limbo de superfície abaxial vinosa com máculas escuras, venação não reticulando em direção ao ápice, nervuras impressas; pétalas de 9 a 10

4. Nymphaea lingulata

1.1. Nymphaea amazonum Mart. \& Zucc., Abh. Math.-Phys. Cl. Konigl. Bayer. Akad. Wiss.1: 363364. 1832.

Fig. 1a-c

Folhas flutuantes, anel de tricomas no ápice do pecíolo; limbo 7-14 × 6-12 cm, oval a elíptico, ápice arredondado, margem inteira, nervuras radiais reticulando fracamente a proeminentemente em direção ao ápice, principais $13-16$, lobos basais $2,8-7,3 \times 3-5,5 \mathrm{~cm}$, face adaxial verde, abaxial vinosa com máculas enegrecidas. Flores de antese noturna; sépalas 4, 3,6-5,5 × 1,7-2,7 cm, ovais, verdes com traços enegrecidos; pétalas $16,3,4-5,5$ $\times 1,3-2,2 \mathrm{~cm}$, ovais, brancas; transição pétala-estame gradual; estames 96-116, creme, externos petalóides 17-22 × 8-12 mm, internos filiformes 9-13 × $1 \mathrm{~mm}$, anteras com apêndices ca. $1 \mathrm{~mm}$; carpelos 22-27, completamente unidos, apêndices carpelares 7-9 $\mathrm{mm}$, clavados, creme, às vezes com o ápice rosado. Frutos 4-6,7 cm diâm.; sementes ovóides ca. $1 \mathrm{~mm}$. Material selecionado: Araruama. Engenho Grande, rua transversal a Estrada do Engenho Grande, alagado, 21.IV.1999, C.B. Moreira 43 (R). Armação de Búzios. Pântano da Malhada, Bairro Vila Verde, 6.III.2004, M. Vianna Filho 60 (R). Bom Jesus de Itabapoana. alagado no canteiro de obras, 4.III.2008, A. Moreira \& M. Gabriel 101 (R). Cabo Frio. Praia de Tucuns, na lagoa, 22.IX.1987, D. Araújo 8164 (GUA). Carapebus. Lagoa Paulista, 31.VIII.2006, C.P. Bove et al. 1758 (R). Macaé. Lagoa Jurubatiba, 5.IV.1983, D. Araújo 5496 (GUA). Mangaratiba. Restinga de Mangaratiba, Área da Aeronáutica, 3.I.2002, L.F.T. Menezez et al. 855 (RBR). São Pedro dÁldeia. alagado em uma fazenda à beira da Rodovia RJ 106, entre Iguaba e São Pedro, 11.IV.1982, H.Q. Boudet Fernandes 457 (GUA). Silva Jardim. Estrada para Gaviões ca. de $12 \mathrm{~km}$ de Silva Jardim, 19.II.2003, C.P. Bove \& W. Costa $1106(\mathrm{R})$.

Ocorre no México, Panamá, Caribe e América do Sul tropical. No Brasil tem ampla distribuição (Wiersema 1987; Irgang \& Gastal 1996; Pott 1998). No Rio de Janeiro: D30, N30, O29, O30, Q25, S26, S28, T8, T24, T27. Floresce e frutifica durante os meses de janeiro a setembro.

Distingue-se facilmente das outras espécies pelo anel de tricomas no ápice do pecíolo. Possui duas subespécies, contudo no Rio de Janeiro só ocorre $N$. amazonum Mart. \& Zucc. subsp. amazonum, que é caracterizada, entre outros aspectos, por apresentar menos de 200 estames. Conhecida pelo nome popular de batata-d'água ou lírio-d'água, assim como as várias espécies de Nymphaea de pétalas brancas. Ilustrações desta espécie são encontradas em Wiersema (1987: 83) e Bove \& Paz (2009: 89). 

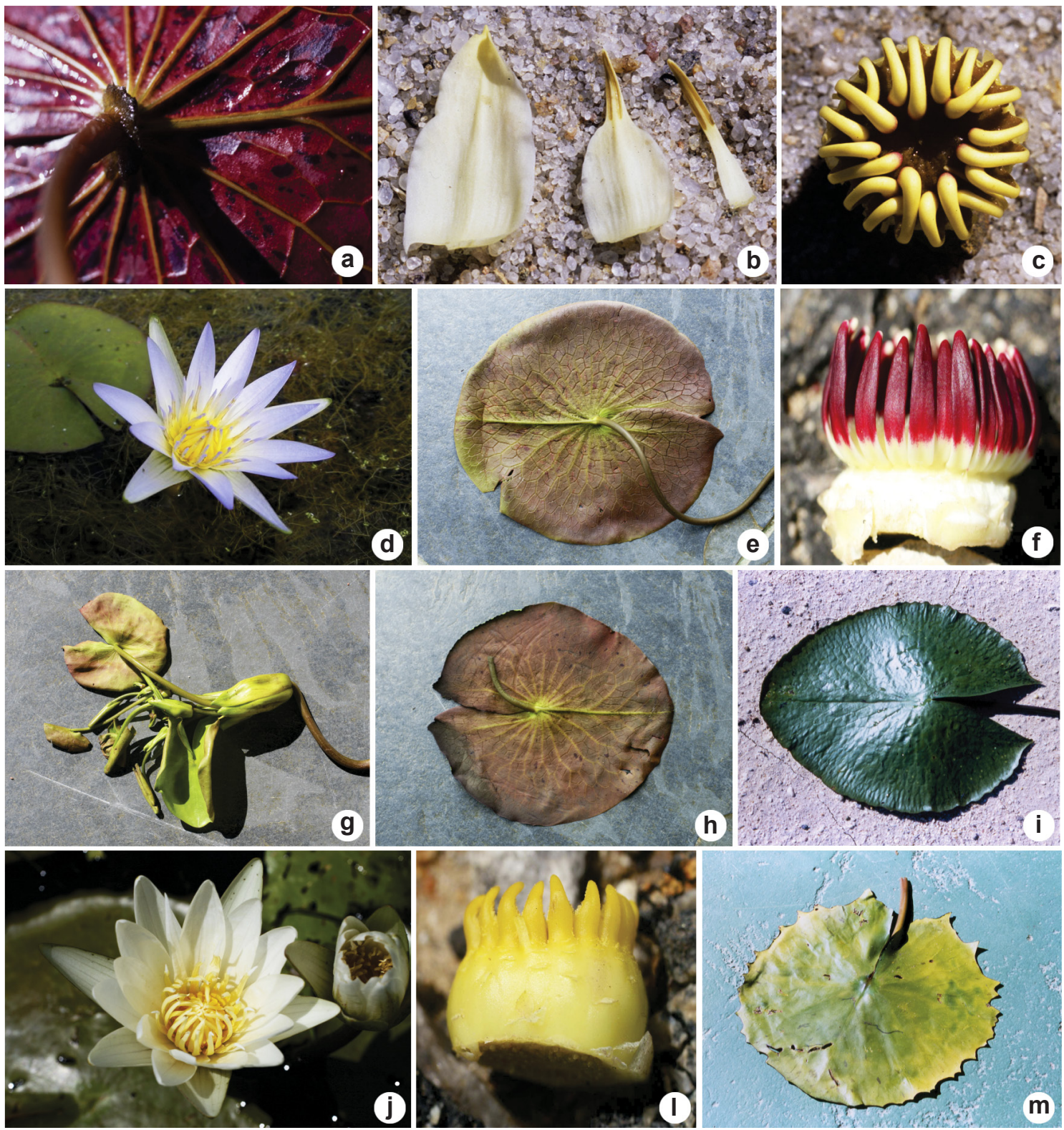

h

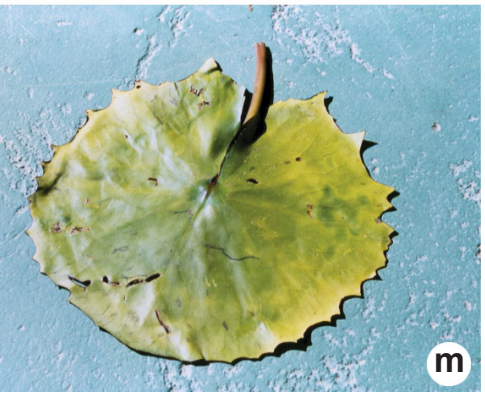

Figura 1 - a-c. Nymphaea amazonum - a. anel de tricomas no ápice do pecíolo; b. gradação pétala-estame; c. apêndices carpelares clavados (Bove 1921). d. Nymphaea caerulea -d. flor (Santos 143). e-g. Nymphaea lasiophylla-e. superfície abaxial da folha; f. apêndices carpelares lingulados; g. flores tubulíferas (Bove 2188). h. Nymphaea lingulata $-\mathrm{h}$. face abaxial da folha (Bove 1939). i-k. Nymphaea pulchella - i. face adaxial da folha; j. flor; k. apêndices carpelares triangulares. (Bove 1588). 1. Nymphaea rudgeana - 1. face adaxial da folha (Bove 1221). Fotos: C.P. Bove; d I.E.Santo. Figure 1 - a-c. Nymphaea amazonum - a. ring of pubescence at apex of petiole; b. gradation petals-stamens; c. clavate carpellary appendages (Bove 1921). d. Nymphaea caerulea - d. flower (Santos 143). e-g. Nymphaea lasiophylla - e. abaxial leaf surface; f. lingulate carpellary appendages; g. tubuliferous flowers (Bove 2188). h. Nymphaea lingulata - h. abaxial leaf surface (Bove 1939). i-k. Nymphaea pulchella $-\mathrm{i}$. adaxial leaf surface; j. flower; $\mathrm{k}$. carpellary appendages short-conical. (Bove 1588). 1. Nymphaea rudgeana -1 . adaxial leaf surface (Bove 1221). Photos: C.P. Bove; d I.E.Santo. 
1.2. Nymphaea caerulea Savigny, Ann. Mus. Natl. Hist. Nat.1: 366-371. 1802. Fig. 1d

Folhas flutuantes, ausência anel de tricomas no ápice do pecíolo; limbo 10-16,5-35 ×9-32,5 cm, suborbicular, ápice arredondado, margem inteira a levemente crenada, nervuras radiais proeminentes reticulando em direção ao ápice, principais 17-21, lobos basais (4)5-15,6 × (4,3)6,5-15,5 cm, face adaxial verde, face abaxial verde-vinosa com máculas avermelhadas. Flores diurnas; sépalas 4, 7-9,3 × 1,5-2,7 cm, oval-lanceoladas, verdes com linhas enegrecidas; pétalas (9)15-20, 4,1-9 $\times$ 1,3-2,5 cm, oval-lanceoladas, base branca ou amarela e ápice lilás a lilás-azulado; transição pétala-estame abrupta; estames 53-87 amarelos, filiformes externos muito maiores (28)37-43 $\times 2 \mathrm{~mm}$, internos 14-18 × $1 \mathrm{~mm}$, anteras com apêndices longos, lilás a lilás-azulado; carpelos 13-17, parcialmente unidos, apêndices carpelares 1-2 mm, triangulares, amarelos. Frutos 7,5-8,5 cm diâm., sementes ca. 1,5 mm.

Material examinado: Cachoeiras de Macacu. Reserva Ecológica de Guapiaçu, 11.VII.2008, I.E. Santo et al. 143 (R). Campos dos Goytacazes. Lagoa Feia, Ponta Grossa dos Fidalgos, 11.XII.2004, C.P. Bove et al. 1416 (R). Itatiaia. 20.IV.1962, H.E. Strang 364 (GUA). Macaé. PARNA Restinga de Jurubatiba, segundo braço da Lagoa de Cabiúnas, a cerca de $250 \mathrm{~m}$ do corpo da lagoa, (22017'44,6"S; 4141'14,1"W), 15.VII.2014, F.R.B. Barcelos et al. 74 (R). MAGÉ, Lago Azul, 25.X.1982, S. Sarahyba et al. 254 (GUA). Vassouras. Horto Florestal de Pau Grande, 20.II.1978. M. Santos et al. 09 (RB).

Ocorre em países da África, Estados Unidos e Costa Rica. No Brasil nos estados do Mato Grosso do Sul, Minas Gerais, Espírito Santo, Rio de Janeiro e São Paulo. No Rio de Janeiro: L33, N30, O13, Q20, Q17, P3. Floresce e frutifica durante o ano todo.

Esta espécie é muito semelhante à Nymphaea pulchella DC., porém diferencia-se desta principalmente pela coloração lilás-azulada do ápice das pétalas e folhas de margem inteira a levemente crenada. Nymphaea pulchella apresenta pétalas brancas e folhas irregularmente denteadas. $N$. caerulea tem origem africana, mas ocorre de forma subespontânea nos neotrópicos (Padgett \& Les 2004). Conhecida pelo nome popular de lótus-azul ou flor-de-lis-sagrada. Ilustrações desta espécie são encontradas em Conard (1905: plate 8) e Mendonça \& Bove (2005: 47, 49-52).

1.3. Nymphaea lasiophylla Mart. \& Zucc., Abh. Math.-Phys. Cl. Königl. Bayer. Akad. Wiss. 1:364. 1832.

Fig. 1e-g
Folhas flutuantes, ausência de anel de tricomas no ápice do pecíolo; limbo 12-23 $\times$ 10-20 cm, oval a suborbicular, ápice arredondado, margem inteira, nervuras radiais reticulando fracamente em direção ao ápice, principais 16-18, lobos basais $9 \times 9 \mathrm{~cm}$, superfície adaxial verdevinoso, superfície abaxial verde com máculas vinosas. Flores noturnas; sépalas 4, 2,5-6,3 × 1,2-2,2 cm, ovais, verdes com linhas enegrecidas; pétalas 19-20, 2,2-6 × 1,1-2 cm, ovais, brancas; transição pétala-estame abrupta; estames 67-122 amarelo-claros, filiformes, externos 18-35 $\times$ 3-5,5 mm, internos 6-9 × 1-1,5 mm, anteras com apêndices curtos ca. $0,5 \mathrm{~mm}$, creme; carpelos (15)22-26, completamente unidos, apêndices carpelares 5-8 mm, lingulados, creme com o ápice púrpura. Frutos não observados, sementes ca. $1 \mathrm{~mm}$ (Wiersema 1897).

Material examinado: Campos dos Goytacazes. BR356 a $1 \mathrm{~km}$ de Campos, em alagado à beira da estrada, 31.III.1981, M.B. Casari et al. 523 (GUA).

Material adicional examinado: CEARÁ: Beberibe. várzea direita do rio Choró, CE 004, 4²'12'28,9"S, 38¹3'37,6"W. 12.V.2008, C.P. Bove et al. 1930 (R). BAHIA: BA 156, $20 \mathrm{~km}$ ao sul de Macaúbas, $13^{\circ} 73^{\prime} 10,2^{\prime} \mathrm{S}, 42^{\circ} 36^{\prime} 57,7^{\prime} \mathrm{W}, 3 . \mathrm{II} .2010$, C.P. Bove et al. $2188(\mathrm{R})$.

No Brasil ocorre do Piauí ao Rio de Janeiro. Apenas um registro para o estado (L32), com flores no mês de março.

Espécie rara no estado do Rio de Janeiro, contudo é abundante no Nordeste do país. Encontrada nos domínios fitogeográficos da Mata Atlântica (BFG 2015) e na Caatinga (Moreira $\&$ Bove 2004). Morfologicamente relacionada à Nymphaea lingulata Wiersema, por ambas apresentarem carpelos lingulados; diferenciase desta por possuir cerca do dobro do número de pétalas e flores tubulíferas frequentes. $\mathrm{O}$ único registro desta espécie para o estado é uma unicata com apenas uma flor fechada (sem flores tubulíferas) identificada pelo especialista no grupo (J.H.Wiersema). Frutos raramente se desenvolvem (Wiersema 1987). Ilustrações desta espécie são encontradas em Wiersema (1987: 92).

\subsection{Nymphaea lingulata Wiersema, Brittonia 36(3): 215.1984. \\ Fig. 1h}

Folhas flutuantes, ausência de anel de tricomas no ápice do pecíolo; limbo 10-21 ×9,5$18,5 \mathrm{~cm}$, oval a suborbicular, ápice arredondado, margem inteira, nervuras radiais, impressas, não reticulando em direção ao ápice, principais 16-18, lobos basais 3,5-8 × 4,2-8,5 cm, superfície adaxial 
verde, superfície abaxial vinosa com máculas escuras. Flores noturnas; sépalas 4, 5,4-6 × 1,8-2,2 $\mathrm{cm}$, ovais, verdes com linhas enegrecidas; pétalas 9-10, 5,1 × 1,3-1,9 cm, ovais, alvas; transição pétala-estame abrupta; estames 86-87, creme de base púrpura, externos petalóides 29-35 × 4-6 mm, internos filiformes 18-19 × 1-2 mm, anteras com apêndices curtos ca. $1 \mathrm{~mm}$, creme; carpelos 21-22, completamente unidos, apêndices carpelares (4)6-8 $\mathrm{mm}$, lingulados, creme de base púrpura. Frutos 2,5-3 cm diâm., sementes ovóides ca. $1 \mathrm{~mm}$.

Material examinado: Macaé. Lagoa de Jurubatiba, 28.IV.2009, C.P. Bove \& A. Moreira 1939 (R).

Material adicional examinado: GOIÁS: Estrada Mozarlândia-Nova-Crixás a $32,9 \mathrm{~km}$ do trevo, 15.XI.1999, C.P. Bove et al. 612 (R). PERNAMBUCO: Ilha de Itamaracá (PE-35) próximo da estrada da Baía dos Golfinhos, $7^{\circ} 15^{\prime} 55,2^{\prime \prime S}, 34^{\circ} 51^{\prime} 42,3^{\prime \prime} \mathrm{W}, 17$. VII.2001, C. P. Bove et al. $878(\mathrm{R})$.

Ocorre na Bolívia, Paraguai e Brasil nos estados: Maranhão, Ceará, Pernambuco, Alagoas, Goiás, Minas Gerais, Mato Grosso do Sul e Rio de Janeiro: O29. Coletada com flores no mês de abril.

Espécie de ocorrência rara no estado do Rio de Janeiro, com apenas um registro no município de Macaé, mesmo após várias tentativas de coleta. Pode ser confundida com Nymphaea lasiophylla por ambas apresentarem carpelos lingulados, contudo diferencia-se desta principalmente pelo menor número de pétalas, superfície abaxial da folha vinosa, não reticulando em direção ao ápice da folha e ausência de flores tubulíferas. Nervuras impressas mais patentes em material herborizado (Pott \& Pott 2000). Flor possui odor forte e adocicado característico. Ilustrações desta espécie são encontradas em Wiersema (1987: 95) e Bove \& Paz (2009: 91).

1.5. Nymphaea pulchella DC., Syst. Nat., 2: 51. 1821.

Fig. 1i-1

Nymphaea ampla (Salisb.) DC., Syst. Nat., 2: 54. 1821.

Folhas flutuantes, ausência anel de tricomas no ápice do pecíolo; limbo 10-16,5-35 × 9-32,5 $\mathrm{cm}$, suborbicular, ápice arredondado, margem sinuoso-denteada a denteada, nervuras radiais proeminentes reticulando em direção ao ápice, principais $17-21$, lobos basais (4)5-15,6 $\times$ $(4,3) 6,5-15,5 \mathrm{~cm}$, face adaxial verde, face abaxial verde-vinosa com máculas púrpuras. Flores diurnas; sépalas 4, 7-9,3 × 1,5-2,7 cm, ovallanceoladas, verdes com linhas enegrecidas; pétalas (9)15-20, 4,1-9 × 1,3-2,5 cm, oval-lanceoladas, alvas; transição pétala-estame abrupta; estames 53-87 amarelos, filiformes, externos muito maiores (28)37-43 × $2 \mathrm{~mm}$ que internos 14-18 × $1 \mathrm{~mm}$, anteras com apêndices longos, alvos; carpelos 13-17, parcialmente unidos, apêndices carpelares 1-2 mm, triangulares, amarelos. Frutos 7,5-8,5 cm diâm., sementes ca. 1,5 mm.

Material selecionado: Araruama. Engenho Grande, rua transversal à Estrada do Engenho Grande, 20.XII.1998, C.B. Moreira 33 (R). Aramação dos Búzios. Estrada Búzios - Cabo Frio, a $500 \mathrm{~m}$ do trevo de Búzios, 1.VII.2001, C.P. Bove \& W. Costa 871 (R). Cabo Frio. alagado atrás da praia da Ferradura, 22.XII.1982, M.B. Casari et al. 910 (GUA). Campos dos Goytacazes. Lagoa Feia, Canto do Toco, 17.V.1983, D. Araújo \& N.C. Maciel 5582 (GUA). Carapebus. Lagoa de Carapebus, 11.V.1999, C.P. Bove et al. 431 (R). Itaguaí. 28.IV.1963, C. Pereira \& E. Pereira $66(\mathrm{HB})$. Macaé. Restinga de Jurubatiba, Lagoa de Jurubatiba, 8.XI.2008, F.S.M. de Mattos 01 (RFA). Magé. Magé-Manilha, Estrada do Contorno, aprox. $5 \mathrm{~km}$ após a Fazenda Sendas, lado direito em direção a Manilha-Rio, 2.IX.1998, D.G. Mantuano \& R.M. Lisboa 03 (R). Quissamã. alagado na estrada indo para a Lagoa Pires, 18.IX.2008, A. Moreira et al. 126 (R). Rio de Janeiro. Av. das Américas, em frente à Pedra de Itaúna, 20.VIII.1980, M.B. Casari et al. 320 (GUA). Sapucaia. Fazenda Pilatos, $22^{\circ} 03$ '07'S, 4249'33"W, 480 m, 22.IX.2000, F.B. Ferreira 43/35 (RFA). Saquarema. Restinga de Itaúna, 7.II.1973. $C$. Farney 234 (RB). Silva Jardim. Juturnaíba, Rio São João, 12.V.1976, M.C. Viana \& R.F. Oliveira 697 (GUA).

Ocorre do México ao Brasil. No Brasil nos seguintes estados: Pará, Ceará, Pernambuco, Alagoas, Sergipe, Bahia, Minas Gerais, Rio de Janeiro, Santa Catarina e Rio Grande do Sul. No Rio de Janeiro: L20, L32, M32, M33, N30, N31, O30, Q24, R17, S27, S28, T23, T24, U7 e U13. Floresce e frutifica durante o ano todo.

A margem das folhas apresenta variação de acordo com o seu estágio de desenvolvimento, sendo as mais jovens inteiras a levemente sinuosodenteadas e as mais desenvolvidas denteadas. Wiersema et al. (2008) distinguem N. pulchella de $N$. ampla (Salisb.) DC. pelo menor número de pétalas, estames e carpelos; folhas de margens sinuoso-denteadas com dentes obtusos; máculas púrpura e venação da folha fracamente reticulada (em oposição às folhas de margem fortemente denteada, com dentes agudos, máculas pretas e venação fortemente reticulada). A distribuição geográfica de ambas se sobrepõe em parte das Antilhas, México e América Central; no entanto apenas $N$. pulchella ocorre na América do Sul. Ilustrações desta espécie são encontradas em Conard (1905: plate 5) e Bove \& PAZ (2009: 93). 
1.6. Nymphaea rudgeana G. Meyer, Prim. Fl. Esseq. 198. 1818.

Fig. $1 \mathrm{~m}$

Folhas flutuantes, ausência anel de tricomas no ápice do pecíolo; limbo $14-26 \times 12,8$ $26,8 \mathrm{~cm}$, suborbicular, ápice arredondado, margem irregularmente denteada, nervuras radiais proeminentes reticulando em direção ao ápice, principais $18-19$, lobos basais $6-10 \times 6,3-12,6$ $\mathrm{cm}$, face adaxial verde com máculas avermalhadas (às vezes enegrecidas), face abaxial vinosa. Flores noturnas; sépalas 4, 3,6-6,6 × 1,7-3,2 cm, ovais, verdes com linhas enegrecidas; pétalas $16-20,5 \times 2,5-2,6 \mathrm{~cm}$, ovais, alvas; transição pétala-estame gradual, estames $64-124$, creme a levemente amarelados, externos petalóides 27-35 $\times 12-20 \mathrm{~mm}$, internos filiformes $12-15 \times 1 \mathrm{~mm}$, anteras com apêndices curtos ca. $1 \mathrm{~mm}$, creme; carpelos 16-25, completamente unidos, apêndices carpelares 4-7 mm, clavados, creme a levemente amarelados. Frutos 4 cm diâm., sementes ca. 1 $\mathrm{mm}$.

Material selecionado: Campos dos Goytacazes. Lagoa Feia, Ponta Grossa dos Fidalgos, 11.XII.2004, C.P. Bove et al. 1407 (R). Carapebus. Lagoa Comprida, 20.X.2003, C.P. Bove et al. 1221 (R). Casimiro de Abreu. Lagoa Iodada, 13.III.1981, M.B. Casari 500 (GUA). Itaboraí. Rio Guaraí, 29.XI.1976. D. Araújo 1371 (GUA). Macaé. Lagoa de Jurubatiba, 5.IV.1983, M.B. Casari \& D.S.D. Araújo 977 (GUA). Quissamã. Lagoa Amarra-Boi, 30.IV.2009, C.P. Bove et al. 1993 (R). Rio de Janeiro. Lagoa da Tijuca, 28.IX.1872, Glaziou 1258.

Ocorre na América Central, Antilhas, norte e leste da América do Sul. Tem ampla distribuição no Brasil. No Rio de Janeiro: L32, M32, M33, N30, N31, O30, Q26, R18 e U13. Floresce e frutifica nos meses de abril a outubro.

Caracteriza-se por apresentar folhas de margem profundamente denteada e carpelos clavados. Flor possui odor forte (lembrando éter ou acetona). Ilustrações desta espécie são encontradas em Wiersema (1987: 98) e Bove \& Paz (2009: 95).

\section{Agradecimentos}

Aos curadores e equipe dos herbários GUA, HB, R, RB, RFA e RBR. Ao Inaldo do Espírito Santo, a foto de $N$. caerulea. Ao Conselho Nacional de Desenvolvimento Científico e Tecnológico (Edital PROTAX, Processo 562251/2010-3), a bolsa de Produtividade de C.P.B. Ao Instituto Chico Mendes de Conservação da Biodiversidade, as licenças de coleta concedidas.

\section{Referências}

BFG. 2015. Growing knowledge: an overview of seed plant diversity in Brazil. Rodriguésia 66: 1085 1113.

Bove, C.P. 2002. A família Nymphaeaceae no estado do Rio de Janeiro. Habitat 72: 45-48.

Bove, C.P. \& Paz, J. 2009. Guia de campo das plantas aquáticas do Parque Nacional da Restinga de Jurubatiba, Rio de Janeiro, Brasil. Museu Nacional, Rio de Janeiro. 175p. (Série livros, 35).

Caspary, R. 1878. Nymphaeaceae. In: Martius, C.P.F.; Eichler, A.W. \& Urban, I. (eds.). Flora brasiliensis. Lipsiae, Frid. Fleischer. Vol. 4, pars 2, pp. 131-184, tab. 28-39.

Conard, H.S. 1905. The waterliles: a monograph of the genus Nymphaea. The Carnegie Institution of Washington, Washington. 279p.

Duke, J.A. 1963. Nymphaeaceae. In: Woodson, R.F. \& Schery, R.W. Flora do Panama. Annals of the Missouri Botanical Garden 49: 137-143.

Feres, F. \& Amaral, M.C.E. 2003. Nymphaeaceae. In: Wanderley, M.G.L.; Shepherd, G.J.; Melhem, T.S.; Giulietti, A.M. \& Kirizawa, M. (org.). Flora fanerogâmica do estado de São Paulo. Instituto de Botânica, São Paulo. Vol. 3, pp. 241-245.

Irgang, B.E. \& Gastal Jr., C.V.S. 1996. Macrófitas aquáticas da planície costeira do RS. Edição dos autores, Porto Alegre. 290p.

Löhne, C.; Borsch, T. \& Wiersema, J.H. 2007. Phylogenetic analysis of Nymphaeales using fast-evolving and noncoding chloroplast markers. Botanical Journal of the Linnean Society 154: 141-163.

Mendonça, R.M. \& Bove, C.P. 2005. O lírio azul do Nilo em águas brasileiras. Habitat 77: 47-52.

Moreira, A.D.R. \& Bove, C.P. 2004. Plantas aquáticas da Caatinga - um paradoxo? Habitat 77: 38-49.

Padget, D.J. \& Les, D.H. 2004. Nymphaeaceae. In: Smith, N.; Mori, S.A.; Henderson, A.; Stevenson, D.W. \& Heald, S.V. (eds.). Flowering plants of the neotropics. Princeton University Press, Princeton. Pp. 271-273.

Pott, V.J. 1998. A família Nymphaeaceae no Pantanal, Mato Grosso e Mato Grosso do Sul, Brasil. Acta Botanica Brasilica 12: 183-194.

Pott, V.J. \& Pott, A. 2000. Plantas aquáticas do Pantanal. Embrapa, Brasília. 404p.

Sucre, B.D. 1959. Nymphaeaceae da cidade do Rio de Janeiro. Rodriguésia 21-23: 239-242.

Wiersema, J.H. 1987. A monograph of Nymphaea subgenus Hydrocallis (Nymphaeaceae). Systematic Botany Monographs 16: 1-112.

Wiersema, J.H.; Novelo, A.R. \& Bonilla-Barbosa, J.R. 2008. Taxonomy and typification of Nymphaea ampla (Salisb.) DC. sensu lato (Nymphaeaceae). Taxon 57: 967-974. 


\section{Lista de exsicatas}

Andrade, Z. s.n. (1.2). Araújo, D. 1371 (1.6), 3038 (1.5), 3822 (1.6), 3823 (1.2), 3824 (1.5), 4056 (1.6), 4057 (1.5), 4519 (1.5), 5496 (1.1), 5497 (1.5), 5521 (1.5), 5582 (1.5), 8164 (1.1), 8165 (1.5). Bove, C.P. 122 (1.5), 126 (1.5), 431 (1.5), 450 (1.5), $478(1.5), 612$ (1.4), 871 (1.5), 873 (1.5), 878 (1.4), 1106 (1.1), 1221 (1.6), 1407 (1.6), 1416 (1.2), 1530 (1.5), 1561 (1.5), 1588 (1.5), 1595 (1.6), 1752 (1.1), 1758 (1.1), 1921 (1.1), 1993 (1.6), 1930 (1.3), 1939 (1.4), 1989 (1.6), 2188 (1.3). Boudet-Fernandes, H.Q. 457 (1.1). Casari, M. В. 320 (1.5), 468 (1.2), 470 (1.6), 473 (1.5), 496 (1.5), 500 (1.6), 507 (1.6), 509 (1.6), 523 (1.3), 526 (1.5), 547 (1.5), 645 (1.5), 646 (1.2), 741 (1.2), 746 (1.5), 910 (1.5), 977 (1.6). Contaifer, R.B. 05 (1.5). Costa, A. 579 (1.5). Dimitri, L. 3711 (1.5). Farney, C. 234 (1.5). Ferreira, F.B. 43/35 (1.5). Galvão, R. 680 (1.6). Giordano, L.C. 251 (1.2). Glaziou 1258 (1.6). Gil, A. 15 (1.1). Maas, P.J.M. 3147 (1.5). Mantuano, D.G. 03 (1.5). Matos, M. 4454 (1.5). Matos, P.M. 63 (1.5). Mattos, F.S.M. 01 (1.5). Mendonça, P.P. 01 (1.5). Menezez, L.F.T. 855 (1.1). Moreira, A. 69 (1.5), 101 (1.1), 117 (1.5), 119 (1.1), 126 (1.5). Moreira, C.B. 33 (1.5), 37 (1.5), 43 (1.1), 44 (1.5). Moura, R. 49 (1.5), 159 (1.5). Paz, J. 392 (1.1). Pereira, C. 66 (1.5). Rangel-Schwacke: s.n. (1.5). Sampaio, A.J. s.n. (1.5). Santo, I.E. 143 (1.2). Santos, M. 09 (1.2). Sarahyba, S. 254 (1.2). Segadas-Vianna 1307 (1.1). Smith, L.B. 6647 (1.1), 6648 (1.5). Strang, H.E. s.n. (1.5), 364 (1.2). Souza, M.C. 258 (1.1). Viana, M.C. 697 (1.5). Vianna-Filho, M. 60 (1.1). Zippin, M. s.n. (1.6), 06 (1.5). 